\title{
A priori error analysis of stabilized mixed finite element method for reaction-diffusion optimal control problems
}

Hongfei Fu ${ }^{1 *}$ (D), Hui Guo', Jian Hou² and Junlong Zhao'

\section{*Correspondence:}

hongfeifu@upc.edu.cn

${ }^{1}$ College of Science, China

University of Petroleum, Qingdao,

266580, China

Full list of author information is

available at the end of the article

\section{Springer}

\begin{abstract}
In this paper, we propose a novel stabilized mixed finite element method for the approximation of optimal control problems governed by reaction-diffusion equations. Compared with the classical mixed finite element methods, the main contributions of this paper are as follows. First, the novel method uses only one stabilization parameter which is not mesh-dependent, and, the new mixed bilinear formulation is coercive and continuous. Second, the novel method is easy to be implemented on a computer using the standard Lagrange finite element. Third, the solutions of the novel method to the optimal control problems require low regularities. Fourth, the Ladyzhenkaya-Babuska-Brezzi (LBB) or the inf-sup condition for the mixed element spaces is unnecessary. Based on the novel method, we derive both continuous and discrete optimality systems for the corresponding constrained optimal control problems, and then a priori error analysis in a weighted norm is discussed. Finally, numerical experiments are given to confirm the efficiency and reliability of the novel stabilized method.
\end{abstract}

Keywords: optimal control; reaction-diffusion equation; stabilized mixed finite element; a priori error analysis; numerical experiments

\section{Introduction}

Optimal control problems and their finite element solutions are attracting increasingly attentions of scientists and engineers. For systematic introductions of finite element methods and their applications in solving optimal control problems, we refer the reader to [1-6].

In this paper, we are interested in the following distributed type optimal control problem:

$$
\min _{u \in U_{a d}} \frac{1}{2} \int_{\Omega}\left(y-y_{d}\right)^{2}+\frac{1}{2} \int_{\Omega}\left(\sigma-\sigma_{d}\right)^{2}+\frac{\gamma}{2} \int_{\Omega} u^{2}
$$

subject to a first-order mixed type reaction-diffusion equation

$$
\begin{cases}\operatorname{div} \sigma+c y=f+\mathcal{B} u, & \text { in } \Omega, \\ \sigma+\mathcal{A} \nabla y=0, & \text { in } \Omega,\end{cases}
$$

(c) $2016 \mathrm{Fu}$ et al. This article is distributed under the terms of the Creative Commons Attribution 4.0 International License (http://creativecommons.org/licenses/by/4.0/), which permits unrestricted use, distribution, and reproduction in any medium, provided you give appropriate credit to the original author(s) and the source, provide a link to the Creative Commons license, and indicate if changes were made. 
and combined with homogeneous Dirichlet boundary condition

$$
y=0, \quad \text { on } \partial \Omega .
$$

Here $\gamma>0$ is a penalty parameter, it is used to measure the relative importance of the terms appearing in the definition of the cost functional; $\mathcal{B}$ is a linear operator; $U_{a d}$ is an admissible convex control set. Detailed information as regards this problem in a functional analysis setting will be discussed later.

This model problem plays an important role in many scientific and engineering applications. For example, it can represent an optimal control of Darcy flows, where the primal state variable $y$ means the pressure, the flux state variable $\sigma$ stands for the velocity field, and the control variable $u$ is an external force. A priori error estimates of finite element approximations for optimal control problems governed by linear elliptic equations were studied extensively; see, for example, Refs. [5, 7-10]. Recently, mixed finite element method has also been found very useful in solving such optimal control problems which contain the flux state variable, see Refs. [11-16]. But all these works need a matched mixed finite element spaces for the state variable and its flux, i.e., LBB stability condition must be strictly satisfied. One of their best choices is the Raviart-Thomas (RT) element, see [17], and therefore, the widely used Lagrange elements are excluded.

Inspired by the idea of so-called Galerkin least squares method, proposed by Hughes et al. [18] and theoretically analyzed by Franca and Stenberg [19], and also the idea of so-called unusual stabilized finite element method [20, 21] for advection-diffusion equations, we propose here a first-order mixed type stabilized finite element method for the elliptic optimal control problems. The novel method is proved to be efficient and reliable both in theoretical error analysis and numerical tests. It also embodies some advantages compared with the former work [22]: It uses only one stabilization parameter which is not mesh-dependent, however, the corresponding mixed bilinear formulation is still coercive and continuous; it is easier to be implemented using the standard Lagrange finite elements; it has relatively lower regularity requirements of the solutions to optimal control problems; and it can be extended to solve optimal control problems governed by other type partial differential equations. Furthermore, compared with the classical mixed finite element method, it also has a free choice of mixed element spaces without the requirement of LBB stability condition, and less degree of freedoms (DoFs) are adopted. Thus, it is more competitive in the practical computation and especially in the high-dimensional case.

The rest of the paper is organized as follows: In Section 2, we first propose the novel stabilized method which uses only one stabilization parameter. Then some preliminary results are presented and an optimality system at the continuous level is deduced for the optimal control problem. In the end of this section, explicit solutions of different cases of the admissible control set are discussed. In Section 3, discretization using standard Lagrange element is discussed, and discrete optimality system is also derived. In Section 4, an optimal-order weighted norm error analysis with a low regularity requirement for the optimal control problem is discussed. In Section 5, we conduct some numerical experiments to verify the theoretical analysis. In the last section, some concluding remarks are given. 


\section{Optimal control problems and optimality system}

Let $\Omega$ be a bounded domain in $\mathbb{R}^{d}(d \leq 3)$ with Lipschitz boundary $\partial \Omega$. Let $\mathcal{T}^{h}$ be a family of regular triangulation of $\Omega$, such that $\Omega=\bigcup_{T \in \mathcal{T}^{h}} T$. Denote $h_{T}$ the diameter of the element $T$ in $\mathcal{T}^{h}$, and set $h=\max _{T \in \mathcal{T}^{h}} h_{T}$. In this paper, we shall employ the usual notion for Lebesgue and Sobolev spaces; see Ref. [1] for details. Throughout, let $C$ denote a strictly positive generic constant, not necessarily the same at each occurrence, but always independent of the mesh size $h$.

To give a detailed description of the model optimal control problem, let us define the state spaces

$$
Y=H_{0}^{1}(\Omega), \quad \Sigma=L^{2}(\Omega)^{d} .
$$

and the control space

$$
U=L^{2}(\Omega)
$$

Furthermore, let $U_{a d}$ be a closed convex subset of the control space $U$. In the following context, we will discuss some different cases on the choice of $U_{a d}$.

In the following, we are ready to propose a simple but stable mixed numerical method. To this aim, we assume $\mathcal{A}=\left(a_{i, j}(x)\right)_{d \times d}$ is symmetric and positive definite, and there exist positive constants $\alpha$ and $\beta$ such that

$$
\alpha|\mathcal{X}|^{2} \leq \mathcal{X}^{T} \mathcal{A} \mathcal{X} \leq \beta|\mathcal{X}|^{2}, \quad \forall \mathcal{X} \in \mathbb{R}^{d}
$$

Besides, suppose $c=c(x)$ is bounded below and up by two constants $c_{*}$ and $c^{*}$ such that

$$
0 \leq c_{*} \leq c \leq c^{*}
$$

We now first revisit the classical mixed formulation of problem (1.2)-(1.3): Find $(y, \sigma) \in$ $L^{2}(\Omega) \times H(\operatorname{div} ; \Omega)$ such that for any $(v, \tau) \in L^{2}(\Omega) \times H(\operatorname{div} ; \Omega)$

$$
\left(\mathcal{A}^{-1} \sigma, \tau\right)-(y, \operatorname{div} \tau)+(\operatorname{div} \sigma, v)+(c y, v)=(f+\mathcal{B} u, v) .
$$

Then followed by integration by parts, and inspired by the stabilized finite element method [18-20], we propose a novel stabilized mixed weak formulation for (1.2)-(1.3): find $(y, \sigma) \in Y \times \Sigma$ such that

$$
\mathcal{A}_{\delta}(y, \sigma ; v, \tau)=(f+\mathcal{B} u, v), \quad \forall(v, \tau) \in Y \times \Sigma,
$$

where the mixed bilinear formulation

$$
\begin{aligned}
\mathcal{A}_{\delta}(y, \sigma ; v, \tau)= & \left(\mathcal{A}^{-1} \sigma, \tau\right)+(\nabla y, \tau)-(\sigma, \nabla v)+(c y, v) \\
& -\sum_{T \in \mathcal{T}^{h}} \delta_{T}\left(\mathcal{A}^{-1} \sigma+\nabla y, \tau-\mathcal{A} \nabla v\right)_{T} .
\end{aligned}
$$

Here $\delta_{T}$ is an elementwise mesh-free constant parameter, and $(\cdot, \cdot)_{T}$ denotes the inner product in $L^{2}(T)^{d}$. 
Define the corresponding stabilization norm

$$
\|\{y, \sigma\}\|_{\delta}^{2}=\left(\mathcal{A}^{-1} \sigma, \sigma\right)+\sum_{T \in \mathcal{T}^{h}} \delta_{T}(\mathcal{A} \nabla y, \nabla y)_{T}+(c y, y) .
$$

Then, we can easily derive the following coercive and bounded results.

Proposition 2.1 (Coercivity and boundedness) For $0<\delta_{0} \leq \delta_{T} \leq \delta_{1}<1$, we have

$$
\mathcal{A}_{\delta}(y, \sigma ; y, \sigma) \geq c_{\delta}\|\{y, \sigma\}\|_{\delta}^{2}, \quad \forall(y, \sigma) \in Y \times \Sigma
$$

Moreover, for all $(y, \sigma ; v, \tau) \in(Y \times \Sigma)^{2}$

$$
\mathcal{A}_{\delta}(y, \sigma ; v, \tau) \leq C_{\delta}\|\{y, \sigma\}\|_{\delta}\|\{v, \tau\}\|_{\delta} .
$$

Here $\delta_{i}(i=0,1)$ are two positive constants, $c_{\delta}=1-\delta_{1}$ and $C_{\delta}=\max \left\{4,2+1 / \delta_{0}\right\}$.

Proof On the one hand, it follows from the definition that

$$
\begin{aligned}
& \mathcal{A}_{\delta}(y, \sigma ; y, \sigma) \\
& \quad=\left(\mathcal{A}^{-1} \sigma, \sigma\right)+(c y, y)-\sum_{T \in \mathcal{T}^{h}} \delta_{T}\left(\mathcal{A}^{-1} \sigma, \sigma\right)_{T}+\sum_{T \in \mathcal{T}^{h}} \delta_{T}(\mathcal{A} \nabla y, \nabla y)_{T} .
\end{aligned}
$$

Hence, for $\delta_{T} \leq \delta_{1}<1$, the bilinear form $a(\diamond, \diamond ; \diamond, \diamond)$ is coercive on $Y \times \Sigma$, and it satisfies (2.7).

On the other hand, we conclude from (2.5) that

$$
\begin{aligned}
\mathcal{A}_{\delta}(y, \sigma ; v, \tau)= & \left(\mathcal{A}^{-1} \sigma, \tau\right)+(\nabla y, \tau)-(\sigma, \nabla v)+(c y, v) \\
& -\sum_{T \in \mathcal{T}^{h}} \delta_{T}\left(\mathcal{A}^{-1} \sigma, \tau\right)_{T}+\sum_{T \in \mathcal{T}^{h}} \delta_{T}(\sigma, \nabla v)_{T} \\
& -\sum_{T \in \mathcal{T}^{h}} \delta_{T}(\nabla y, \tau)_{T}+\sum_{T \in \mathcal{T}^{h}} \delta_{T}(\mathcal{A} \nabla y, \nabla v)_{T} .
\end{aligned}
$$

Note that

$$
\sum_{i}\left|a_{i} b_{i}\right| \leq\left(\sum_{i} a_{i}^{2}\right)^{\frac{1}{2}}\left(\sum_{i} b_{i}^{2}\right)^{\frac{1}{2}}
$$

Then for $0<\delta_{0} \leq \delta_{T} \leq \delta_{1}<1$, we have

$$
\begin{aligned}
\mathcal{A}_{\delta}(y, \sigma ; v, \tau) \\
\leq\left[4\left(\mathcal{A}^{-1} \sigma, \sigma\right)+(\mathcal{A} \nabla y, \nabla y)+2 \sum_{T \in \mathcal{T}^{h}} \delta_{T}(\mathcal{A} \nabla y, \nabla y)_{T}+(c y, y)\right]^{\frac{1}{2}} \\
\quad \times\left[4\left(\mathcal{A}^{-1} \tau, \tau\right)+(\mathcal{A} \nabla v, \nabla v)+2 \sum_{T \in \mathcal{T}^{h}} \delta_{T}(\mathcal{A} \nabla v, \nabla v)_{T}+(c v, v)\right]^{\frac{1}{2}}
\end{aligned}
$$




$$
\begin{aligned}
\leq & {\left[4\left(\mathcal{A}^{-1} \sigma, \sigma\right)+\left(2+\frac{1}{\delta_{0}}\right) \sum_{T \in \mathcal{T}^{h}} \delta_{T}(\mathcal{A} \nabla y, \nabla y)_{T}+(c y, y)\right]^{\frac{1}{2}} } \\
& \times\left[4\left(\mathcal{A}^{-1} \tau, \tau\right)+\left(2+\frac{1}{\delta_{0}}\right) \sum_{T \in \mathcal{T}^{h}} \delta_{T}(\mathcal{A} \nabla v, \nabla v)_{T}+(c v, v)\right]^{\frac{1}{2}} \\
\leq & C_{\delta}\|\{y, \sigma\}\|_{\delta}\|\{v, \tau\}\|_{\delta},
\end{aligned}
$$

which proves the boundedness result (2.8).

Proposition 2.2 (Existence and uniqueness) Assume the condition in Proposition 2.1 is valid. Furthermore, let $f \in L^{2}(\Omega)$. Then for given control $u \in L^{2}(\Omega)$, problem (2.4) admits a unique solution $(y, \sigma) \in Y \times \Sigma$.

Proof Proposition 2.1 implies that the mixed bilinear form $\mathcal{A}_{\delta}(\diamond, \diamond ; \diamond, \diamond)$ is coercive and bounded in a weighted norm (2.6). Then the Lax-Milgram lemma implies the existence and uniqueness of the solution pair $(y, \sigma) \in Y \times \Sigma$.

Furthermore, suppose $u=0$ in problem (2.4), we then have the following stability result with respect to the right-hand $\operatorname{term} f$.

Proposition 2.3 (Stability) Let $f \in L^{2}(\Omega)$. If the condition in Proposition 2.1 is valid, then we have

$$
\|\{y, \sigma\}\|_{\delta} \leq C\|f\|_{L^{2}(\Omega)}
$$

where the constant $C$ depends on the Poincaré constant $C_{\Omega}$, and the reciprocal of $c_{\delta}$ and $\delta_{0}$.

Proof Let $(v, \tau)=(y, \sigma)$ in problem (2.4). Then it follows from Proposition 2.1, the Poincaré inequality in Lemma 4.3, and the definition of the stabilization norm that

$$
\begin{aligned}
c_{\delta}\|\{y, \sigma\}\|_{\delta}^{2} & \leq\|f\|_{L^{2}(\Omega)}\|y\|_{L^{2}(\Omega)} \\
& \leq C_{\Omega}\|f\|_{L^{2}(\Omega)}\|\nabla y\|_{L^{2}(\Omega)} \\
& \leq \frac{C_{\Omega}}{\sqrt{\delta_{0}}}\|f\|_{L^{2}(\Omega)}\left(\sum_{T \in \mathcal{T}^{h}} \delta_{T}(\nabla y, \nabla y)_{T}\right)^{1 / 2},
\end{aligned}
$$

which implies the conclusion.

Denote

$$
\mathcal{J}(y, \sigma, u)=\frac{1}{2} \int_{\Omega}\left(y-y_{d}\right)^{2}+\frac{1}{2} \int_{\Omega}\left(\sigma-\sigma_{d}\right)^{2}+\frac{\gamma}{2} \int_{\Omega} u^{2},
$$

where $y=y(u)$ and $\sigma=\sigma(u)$ are $u$-dependent.

Then for the given control set $U_{a d}$, we reformulate the optimal control problem (1.1)(1.3) as follows: (OCP)

$$
\mathcal{J}\left(y^{*}, \sigma^{*}, u^{*}\right)=\min _{u \in U_{a d}} \mathcal{J}(y, \sigma, u)
$$


such that $(y, \sigma, u) \in Y \times \Sigma \times U$ and

$$
\mathcal{A}_{\delta}(y, \sigma ; v, \tau)=(f+\mathcal{B} u, v), \quad \forall(v, \tau) \in Y \times \Sigma .
$$

It then follows from Ref. [3] that the optimal control problem (OCP) has a unique solution $\left(y^{*}, \sigma^{*}, u^{*}\right) \in Y \times \Sigma \times U_{a d}$, and $\left(y^{*}, \sigma^{*}, u^{*}\right)$ is the solution of (OCP) if and only if there is a pair of adjoint state $\left(z^{*}, \omega^{*}\right) \in Y \times \Sigma$, such that $\left(y^{*}, \sigma^{*}, z^{*}, \omega^{*}, u^{*}\right) \in(Y \times \Sigma)^{2} \times U_{a d}$ satisfies the following optimality system: (OS)

State equation:

$$
\mathcal{A}_{\delta}\left(y^{*}, \sigma^{*} ; v, \tau\right)=\left(f+\mathcal{B} u^{*}, v\right), \quad \forall(v, \tau) \in Y \times \Sigma .
$$

Adjoint state equation:

$$
\mathcal{A}_{\delta}\left(v, \tau ; z^{*}, \omega^{*}\right)=-\left(y^{*}-y_{d}, v\right)-\left(\sigma^{*}-\sigma_{d}, \tau\right), \quad \forall(v, \tau) \in Y \times \Sigma .
$$

Optimality condition:

$$
\left(\gamma u^{*}-\mathcal{B}^{*} z^{*}, u-u^{*}\right) \geq 0, \quad \forall u \in U_{a d}
$$

Here $\mathcal{B}^{*}$ is the adjoint operator of $\mathcal{B}$, which satisfies

$$
(\mathcal{B} v, w)=\left(v, \mathcal{B}^{*} w\right), \quad \forall(v, w) \in L^{2}(\Omega) \times H_{0}^{1}(\Omega)
$$

Remark 2.4 For the stabilization parameter $\delta_{T}$ being chosen as a constant $\delta$ in the whole domain $\Omega$, the adjoint states $z^{*}$ and $\omega^{*}$ in (2.14) satisfy the following strong forms:

$$
\begin{cases}-\Delta z=-\left(y-y_{d}\right)-\operatorname{div}\left(\sigma-\sigma_{d}\right), & \text { in } \Omega, \\ z=0, & \text { on } \partial \Omega\end{cases}
$$

and

$$
\omega=\nabla z-\frac{\sigma-\sigma_{d}}{1-\delta}, \quad \text { in } \Omega
$$

In the following, we introduce $z$ and $\omega$ be the solutions of the dual problem such that

$$
\mathcal{A}_{\delta}(v, \tau ; z, \omega)=-(g, v)-(q, \tau), \quad \forall(v, \tau) \in Y \times \Sigma .
$$

Then, similar to the proof in Proposition 2.3, we have the following stability result.

Proposition 2.5 (Stability) Let $g \in L^{2}(\Omega)$ and $q \in L^{2}(\Omega)^{d}$ in problem (2.18). Assume the condition in Proposition 2.1 is valid. Then we have

$$
\|\{z, \omega\}\|_{\delta} \leq C\left(\|g\|_{L^{2}(\Omega)}+\|q\|_{L^{2}(\Omega) d}\right)
$$

where the constant $C$ depends on the Poincaré constant $C_{\Omega}$, and the reciprocal of $c_{\delta}$ and $\delta_{0}$. 
Remark 2.6 Let $f, u^{*} \in L^{2}(\Omega)$. If the condition in Proposition 2.1 is valid, then from Proposition 2.3 we have

$$
\left\|\left\{y^{*}, \sigma^{*}\right\}\right\|_{\delta} \leq C\left(\|f\|_{L^{2}(\Omega)}+\left\|u^{*}\right\|_{L^{2}(\Omega)}\right) .
$$

Furthermore, let $y_{d} \in L^{2}(\Omega)$ and $\sigma_{d} \in L^{2}(\Omega)^{d}$. Then from Proposition 2.5 and the above conclusion we have

$$
\begin{aligned}
\left\|\left\{z^{*}, \omega^{*}\right\}\right\|_{\delta} & \leq C\left(\left\|y^{*}-y_{d}\right\|_{L^{2}(\Omega)}+\left\|\sigma^{*}-\sigma_{d}\right\|_{L^{2}(\Omega)^{d}}\right) \\
& \leq C\left(\|f\|_{L^{2}(\Omega)}+\left\|u^{*}\right\|_{L^{2}(\Omega)}+\left\|y_{d}\right\|_{L^{2}(\Omega)}+\left\|\sigma_{d}\right\|_{L^{2}(\Omega)^{d}}\right) .
\end{aligned}
$$

In the end of this section, we pay special attention on the solution of the variational inequality (2.15). It depends heavily on the structure of the convex set $U_{a d}$. For some cases (see, e.g. $[3,4]$ ), we have the following explicit results.

Case I. $U_{a d}=U$

Then the solution is

$$
u^{*}(x)=\frac{1}{\gamma} \mathcal{B}^{*} z^{*}(x)
$$

Case II. $U_{a d}=\{u \in U: u \geq 0$, a.e. in $\Omega\}$

Then the solution is

$$
u^{*}(x)=\max \left\{0, \frac{1}{\gamma} \mathcal{B}^{*} z^{*}(x)\right\}
$$

Case III. $U_{a d}=\{u \in U: a \leq u \leq b$, a.e. in $\Omega\}$ where the bounds $a, b \in \mathbb{R}$ fulfill $a<b$.

Then the solution is

$$
u^{*}(x)=\max \left\{a, \min \left\{b, \frac{1}{\gamma} \mathcal{B}^{*} z^{*}(x)\right\}\right\} .
$$

Case IV. $U_{a d}=\left\{u \in U: \int_{\Omega} u \geq 0\right\}$

Then the solution is

$$
u^{*}(x)=\max \left\{0,-\frac{1}{\gamma} \overline{\mathcal{B}^{*} z^{*}(x)}\right\}+\frac{1}{\gamma} \mathcal{B}^{*} z^{*}(x)
$$

where $\overline{\mathcal{B}^{*} z^{*}(x)}=\frac{\int_{\Omega} \mathcal{B}^{*} z^{*}(x)}{\int_{\Omega} 1}$.

\section{Stabilized mixed finite element approximation}

In this section, we shall consider the approximation of problem (OCP) based on the novel stabilized mixed finite element method. As the bilinear form $\mathcal{A}_{\delta}(\diamond, \diamond ; \diamond, \diamond)$ is coercive, there is no need to choose the classical mixed element spaces, for example, RT elements [17, 23], as the flux function space. Instead, the widely used Lagrange finite element spaces, for example, the case of piecewise linear elements, can be adopted. 
In this work, we consider the approximations of the state and flux state variables in the following finite element spaces:

$$
\begin{aligned}
& Y^{h}=\left\{v_{h} \in C^{0}(\Omega):\left.v_{h}\right|_{T} \in P_{1}(T), \forall T \in \mathcal{T}^{h}, v_{h}=0 \text { on } \partial \Omega\right\} \subset Y, \\
& \Sigma^{h}=\left\{\tau_{h} \in L^{2}(\Omega):\left.\left(\tau_{h}\right)_{i}\right|_{T} \in P_{1}(T), i=1,2, \ldots, d, \forall T \in \mathcal{T}^{h}\right\} \subset \Sigma .
\end{aligned}
$$

Here $P_{k}$ denotes polynomials of total degree at most $k$.

Furthermore, we consider piecewise constant elements for the approximation of the control variable, that is,

$$
U^{h}=\left\{u_{h} \in U:\left.u_{h}\right|_{T} \in P_{0}(T), \forall T \in \mathcal{T}^{h}\right\}
$$

Let $U_{a d}^{h}=U^{h} \cap U_{a d}$ be the discrete admissible control set. It is apparently so that $U_{a d}^{h} \subset$ $U_{a d}$.

For the finite element spaces defined above, the stabilized mixed finite element approximation of $(\mathrm{OCP})$, which will be labeled as $(\mathrm{OCP})_{h}$, can be described as follows:

$$
\mathcal{J}\left(y_{h}^{*}, \sigma_{h}^{*}, u_{h}^{*}\right)=\min _{u_{h} \in U_{a d}^{h}} \mathcal{J}\left(y_{h}, \sigma_{h}, u_{h}\right)
$$

where $\left(y_{h}, \sigma_{h}, u_{h}\right) \in Y^{h} \times \Sigma^{h} \times U^{h}$ satisfies

$$
\mathcal{A}_{\delta}\left(y_{h}, \sigma_{h} ; v_{h}, \tau_{h}\right)=\left(f+\mathcal{B} u_{h}, v_{h}\right), \quad \forall\left(v_{h}, \tau_{h}\right) \in Y^{h} \times \Sigma^{h} .
$$

It is again well known (see Ref. [3]) that the optimal control problem $(\mathrm{OCP})_{h}$ has a unique solution $\left(y_{h}^{*}, \sigma_{h}^{*}, u_{h}^{*}\right) \in Y^{h} \times \Sigma^{h} \times U_{a d}^{h}$, and that a triplet $\left(y_{h}^{*}, \sigma_{h}^{*}, u_{h}^{*}\right)$ is the solution of $(\mathrm{OCP})_{h}$ if and only if there is a pair of adjoint states $\left(z_{h}^{*}, \omega_{h}^{*}\right) \in Y^{h} \times \Sigma^{h}$, such that $\left(y_{h}^{*}, \sigma_{h}^{*}, z_{h}^{*}, \omega_{h}^{*}, u_{h}^{*}\right) \in$ $\left(Y^{h} \times \Sigma^{h}\right)^{2} \times U_{a d}^{h}$ satisfies the following discrete optimality system: (OS $)_{h}$

State equation:

$$
\mathcal{A}_{\delta}\left(y_{h}^{*}, \sigma_{h}^{*} ; v_{h}, \tau_{h}\right)=\left(f+\mathcal{B} u_{h}^{*}, v_{h}\right), \quad \forall\left(v_{h}, \tau_{h}\right) \in Y^{h} \times \Sigma^{h} .
$$

Adjoint state equation:

$$
\mathcal{A}_{\delta}\left(v_{h}, \tau_{h} ; z_{h}^{*}, \omega_{h}^{*}\right)=-\left(y_{h}^{*}-y_{d}, v_{h}\right)-\left(\sigma_{h}^{*}-\sigma_{d}, \tau_{h}\right), \quad \forall\left(v_{h}, \tau_{h}\right) \in Y^{h} \times \Sigma^{h} .
$$

Optimality condition:

$$
\left(\gamma u_{h}^{*}-\mathcal{B}^{*} z_{h}^{*}, u_{h}-u_{h}^{*}\right) \geq 0, \quad \forall u_{h} \in U_{a d}^{h}
$$

Remark 3.1 The coercivity of the mixed bilinear formulation $\mathcal{A}_{\delta}(\diamond, \diamond ; \diamond, \diamond)$ leads to a positive definite linear algebraic equation in the discrete level, and therefore, the discrete state and adjoint state equations (3.5)-(3.6) can be solved quickly using the popular solvers, such as the conjugate gradient (CG) solver and algebraic multi-grid (AMG) solver.

Let $\mathcal{P}_{h}$ be an $L^{2}$-projection from $U=L^{2}(\Omega)$ to $U^{h}$ such that for any $u \in U$

$$
\left(u-\mathcal{P}_{h} u, \phi\right)=0, \quad \forall \phi \in U^{h} .
$$


It is a matter of calculation that for the optimal control $u^{*} \in U_{a d}$, the projection $\mathcal{P}_{h} u^{*}$ belongs to $U_{a d}^{h}$. For example, we can give a proof for Case IV. In fact, let $\phi \equiv 1 \in U^{h}$ in equation (3.8), then we have

$$
\int_{\Omega} \mathcal{P}_{h} u^{*}=\int_{\Omega} u^{*} \geq 0
$$

which proves the conclusion. The proofs of the other cases are similar. Besides, it is easy to check that

$$
\left.\mathcal{P}_{h} u\right|_{T}=\frac{1}{|T|} \int_{T} u, \quad \forall T \in \mathcal{T}^{h}
$$

where $|T|$ is the area of element $T$.

Finally, similar to the explicit solutions (2.22)-(2.25) to the variational inequality (2.15) for different cases. The solution of (3.7) can also be described explicitly for the corresponding cases; see [3, 4]. We summarize them as below.

Case I. $U_{a d}^{h}=U^{h}$

Then the solution is

$$
u_{h}^{*}=\frac{1}{\gamma} \mathcal{P}_{h}\left(\mathcal{B}^{*} z_{h}^{*}\right)
$$

Case II. $U_{a d}^{h}=\left\{u_{h} \in U^{h}:\left.u_{h}\right|_{T} \geq 0, \forall T \in \mathcal{T}^{h}\right\}$

Then the solution is

$$
u_{h}^{*}=\max \left\{0, \frac{1}{\gamma} \mathcal{P}_{h}\left(\mathcal{B}^{*} z_{h}^{*}\right)\right\}
$$

Case III. $U_{a d}=\left\{u_{h} \in U^{h}: a \leq u_{h} \leq b\right.$, a.e. in $\left.\Omega\right\}$

Then the solution is

$$
u_{h}^{*}=\max \left\{a, \min \left\{b, \frac{1}{\gamma} \mathcal{P}_{h}\left(\mathcal{B}^{*} z_{h}^{*}\right)\right\}\right\} .
$$

Case IV. $U_{a d}=\left\{u_{h} \in U^{h}: \int_{\Omega} u_{h} \geq 0\right\}$

Then the solution is

$$
u_{h}^{*}=\max \left\{0,-\frac{1}{\gamma} \overline{\mathcal{B}^{*} z_{h}^{*}}\right\}+\frac{1}{\gamma} \mathcal{P}_{h}\left(\mathcal{B}^{*} z_{h}^{*}\right)
$$

\section{A priori error estimates}

In this section, we shall give a priori error estimates for the proposed novel stabilized mixed finite element method of optimal control problem.

Before that let us first recall the following interpolation and projection results.

Lemma 4.1 [1] Let $\mathcal{P}_{h}$ be the $L^{2}$-projection defined in (3.8). Then for $u \in H^{1}(\Omega)$, we have

$$
\left\|u-\mathcal{P}_{h} u\right\|_{L^{2}(\Omega)} \leq C h\|u\|_{H^{1}(\Omega)} .
$$


Lemma 4.2 Let $\mathcal{I}_{h}$ be the standard Lagrange interpolation operator defined in Ref. [1]. Then there is a constant $C>0$ such that

$$
\left\|v-\mathcal{I}_{h} v\right\|_{L^{2}(T)}+h_{T}\left|v-\mathcal{I}_{h} v\right|_{H^{1}(T)} \leq C h_{T}^{2}|v|_{H^{2}(T)},
$$

for $\forall v \in H^{2}(T), \forall T \in \mathcal{T}^{h}$.

Lemma 4.3 (Poincaré inequality) There is a positive constant $C_{\Omega}$ which depends only on the domain $\Omega$ such that

$$
\|v\|_{L^{2}(\Omega)} \leq C_{\Omega}\|\nabla v\|_{L^{2}(\Omega)^{d}}, \quad \forall v \in H_{0}^{1}(\Omega) .
$$

In this paper, we aim to demonstrate the following main conclusion between the optimal solutions of (OS) and the stabilized mixed finite element solutions of $(\mathrm{OS})_{h}$.

Theorem 4.4 Suppose that $\left(y^{*}, \sigma^{*}, z^{*}, \omega^{*}, u^{*}\right)$ and $\left(y_{h}^{*}, \sigma_{h}^{*}, z_{h}^{*}, \omega_{h}^{*}, u_{h}^{*}\right)$ are the solutions of (OS) and (OS) $)_{h}$, respectively. Assume that the solutions $\left\{y^{*}, z^{*}\right\} \in H_{0}^{1}(\Omega) \cap H^{2}(\Omega),\left\{\sigma^{*}, \omega^{*}\right\} \in$ $H^{1}(\Omega)^{d}$, and $u^{*} \in H^{1}(\Omega)$. Then there is a positive constant $C$ such that

$$
\begin{gathered}
\left\|u^{*}-u_{h}^{*}\right\|_{L^{2}(\Omega)}+\left\|\left\{y^{*}-y_{h}^{*}, \sigma^{*}-\sigma_{h}^{*}\right\}\right\|_{\delta}+\left\|\left\{z^{*}-z_{h}^{*}, \omega^{*}-\omega_{h}^{*}\right\}\right\|_{\delta} \\
\leq C h\left[\left\|u^{*}\right\|_{H^{1}(\Omega)}+\sum_{v=y, z ; \tau=\sigma, \omega}\left(\left\|v^{*}\right\|_{H^{2}(\Omega)}+\left\|\tau^{*}\right\|_{H^{1}(\Omega)}\right)\right] .
\end{gathered}
$$

Remark 4.5 Compared with Ref. [22], a same optimal-order convergence between the exact solutions and numerical solutions is obtained. However, the requirement of regularities for the flux state $\sigma^{*}$ and adjoint flux state $\omega^{*}$ are both reduced from $H^{2}(\Omega)^{d}$ to $H^{1}(\Omega)^{d}$. This appears to be a more realistic assumption if the original state equation is only $H^{2}$-regular, and if the given data $f, y_{d}, \sigma_{d}$ and the optimal control $u^{*}$ all belong to $L^{2}(\Omega)$. In particular, for Cases I and IV, we can predict that the optimal control $u^{*} \in C^{\infty}(\Omega)$ as if the given data are sufficiently smooth. Indeed, from (2.22) and (2.25) we can observe that the regularity of the optimal control $u^{*}$ agrees with that of the adjoint state $z^{*}$.

To derive the above main result, we introduce $\left(y_{h}\left(u^{*}\right), \sigma_{h}\left(u^{*}\right), z_{h}\left(u^{*}\right), \omega_{h}\left(u^{*}\right)\right) \in\left(Y^{h} \times\right.$ $\left.\Sigma^{h}\right)^{2}$ as the discrete intermediate variables. They are associated with the optimal control solution $u^{*} \in U_{a d}$ and satisfy

$$
\begin{aligned}
& \mathcal{A}_{\delta}\left(y_{h}\left(u^{*}\right), \sigma_{h}\left(u^{*}\right) ; v_{h}, \tau_{h}\right)=\left(f+\mathcal{B} u^{*}, v_{h}\right), \\
& \mathcal{A}_{\delta}\left(v_{h}, \tau_{h} ; z_{h}\left(u^{*}\right), \omega_{h}\left(u^{*}\right)\right)=-\left(y_{h}\left(u^{*}\right)-y_{d}, v_{h}\right)-\left(\sigma_{h}\left(u^{*}\right)-\sigma_{d}, \tau_{h}\right),
\end{aligned}
$$

for any $\left(v_{h}, \tau_{h}\right) \in Y^{h} \times \Sigma^{h}$.

For simplicity of presentation, below let us denote those solutions of (4.5)-(4.6) corresponding to the optimal control $u^{*}$

$$
\left.\widetilde{\gamma}_{h}, \widetilde{\sigma}_{h}, \widetilde{z}_{h}, \widetilde{\omega}_{h}\right) \triangleq\left(y_{h}\left(u^{*}\right), \sigma_{h}\left(u^{*}\right), z_{h}\left(u^{*}\right), \omega_{h}\left(u^{*}\right)\right) .
$$

Now we are ready to prove Theorem 4.4 in three steps. First, we prove a direct result between the intermediate variables and the numerical solutions. 
Lemma 4.6 Let $\left(y_{h}^{*}, \sigma_{h}^{*}, \omega_{h}^{*}, z_{h}^{*}\right)$ and $\left.\widetilde{y}_{h}, \widetilde{\sigma}_{h}, \widetilde{z}_{h}, \widetilde{\omega}_{h}\right)$ be the solutions of (3.5)-(3.6) and (4.5)(4.6), respectively. Then the following estimates hold:

$$
\left\|\left\{y_{h}^{*}-\tilde{y}_{h}, \sigma_{h}^{*}-\widetilde{\sigma}_{h}\right\}\right\|_{\delta}+\left\|\left\{z_{h}^{*}-\widetilde{z}_{h}, \omega_{h}^{*}-\widetilde{\omega}_{h}\right\}\right\|_{\delta} \leq C\left\|u^{*}-u_{h}^{*}\right\|_{L^{2}(\Omega)} .
$$

Proof On the one hand, we conclude from (3.5) and (4.5) that

$$
\mathcal{A}_{\delta}\left(y_{h}^{*}-\tilde{y}_{h}, \sigma_{h}^{*}-\widetilde{\sigma}_{h} ; v_{h}, \tau_{h}\right)=\left(\mathcal{B}\left(u_{h}^{*}-u^{*}\right), v_{h}\right), \quad \forall\left(v_{h}, \tau_{h}\right) \in Y^{h} \times \Sigma^{h}
$$

Selecting $v_{h}=y_{h}^{*}-\tilde{y}_{h}$ and $\tau_{h}=\sigma_{h}-\widetilde{\sigma}_{h}$ in (4.8). It then follows from the stability result in Proposition 2.3 that

$$
\left\|\left\{y_{h}^{*}-\tilde{y}_{h}, \sigma_{h}^{*}-\widetilde{\sigma}_{h}\right\}\right\|_{\delta} \leq C\left\|u^{*}-u_{h}^{*}\right\|_{L^{2}(\Omega)} .
$$

On the other hand, we deduce from (3.6) and (4.6) that

$$
\mathcal{A}_{\delta}\left(v_{h}, \tau_{h} ; z_{h}^{*}-\widetilde{z}_{h}, \omega_{h}^{*}-\widetilde{\omega}_{h}\right)=-\left(y_{h}^{*}-\widetilde{y}_{h}, v_{h}\right)-\left(\sigma_{h}^{*}-\widetilde{\sigma}_{h}, \tau_{h}\right),
$$

for any $\left(v_{h}, \tau_{h}\right) \in Y^{h} \times \Sigma^{h}$.

Let $v_{h}=z_{h}^{*}-\widetilde{z}_{h}$ and $\tau_{h}=\omega_{h}^{*}-\widetilde{\omega}_{h}$. Following the stability result in Proposition 2.5 and the conclusion (4.9), we derive

$$
\left\|\left\{z_{h}^{*}-\widetilde{z}_{h}, \omega_{h}^{*}-\widetilde{\omega}_{h}\right\}\right\|_{\delta} \leq C\left\|\left\{y_{h}^{*}-\tilde{y}_{h}, \sigma_{h}^{*}-\widetilde{\sigma}_{h}\right\}\right\|_{\delta} \leq C\left\|u^{*}-u_{h}^{*}\right\|_{L^{2}(\Omega)} .
$$

Therefore, the proof of Lemma 4.6 is ended.

Then we turn to the validation of an optimal-order convergence between the intermediate variables and the exact solutions.

Lemma 4.7 Let $\left(y^{*}, \sigma^{*}, z^{*}, \omega^{*}\right)$ and $\left.\widetilde{y}_{h}, \widetilde{\sigma}_{h}, \widetilde{z}_{h}, \widetilde{\omega}_{h}\right)$ be the solutions of (2.13)-(2.14) and (4.5)-(4.6), respectively. Furthermore, assume the solutions $\left\{y^{*}, z^{*}\right\} \in H_{0}^{1}(\Omega) \cap H^{2}(\Omega)$ and $\left\{\sigma^{*}, \omega^{*}\right\} \in H^{1}(\Omega)^{d}$. Then the following estimates hold:

$$
\begin{aligned}
& \left\|\left\{y^{*}-\tilde{y}_{h}, \sigma^{*}-\widetilde{\sigma}_{h}\right\}\right\|_{\delta} \leq \operatorname{Ch}\left(\left\|y^{*}\right\|_{H^{2}(\Omega)}+\left\|\sigma^{*}\right\|_{H^{1}(\Omega) d}\right), \\
& \left\|\left\{z^{*}-\widetilde{z}_{h}, \omega^{*}-\widetilde{\omega}_{h}\right\}\right\|_{\delta} \leq C h \sum_{\nu=y, z ; \tau=\sigma, \omega}\left(\left\|v^{*}\right\|_{H^{2}(\Omega)}+\left\|\tau^{*}\right\|_{H^{1}(\Omega)^{d}}\right) .
\end{aligned}
$$

Proof First of all, it is clear that (4.5) is the mixed finite element approximation of (2.13). Therefore, the result (4.12) can easily be proved by the interpolation estimation theory in Lemma 4.2.

Second, we obtain by subtracting (4.6) from (2.14) that

$$
\mathcal{A}_{\delta}\left(v_{h}, \tau_{h} ; z^{*}-\widetilde{z}_{h}, \omega^{*}-\widetilde{\omega}_{h}\right)=-\left(y^{*}-\tilde{y}_{h}, v_{h}\right)-\left(\sigma^{*}-\widetilde{\sigma}_{h}, \tau_{h}\right),
$$

for any $\left(v_{h}, \tau_{h}\right) \in Y^{h} \times \Sigma^{h}$. 
Equivalently, (4.14) can be expressed as

$$
\begin{aligned}
& \mathcal{A}_{\delta}\left(v_{h}, \tau_{h} ; \mathcal{I}_{h} z^{*}-\widetilde{z}_{h}, \mathcal{I}_{h} \omega^{*}-\widetilde{\omega}_{h}\right) \\
& \quad=\mathcal{A}_{\delta}\left(v_{h}, \tau_{h} ; \mathcal{I}_{h} z^{*}-z^{*}, \mathcal{I}_{h} \omega^{*}-\omega^{*}\right)-\left(y^{*}-\tilde{y}_{h}, v_{h}\right)-\left(\sigma^{*}-\widetilde{\sigma}_{h}, \tau_{h}\right),
\end{aligned}
$$

where $\mathcal{I}_{h} z^{*} \in Y^{h}$ and $\mathcal{I}_{h} \omega^{*} \in \Sigma^{h}$ are the standard Lagrange piecewise linear interpolation of $z^{*}$ and $\omega^{*}$, respectively.

Let $v_{h}=\mathcal{I}_{h} z^{*}-\widetilde{z}_{h}$ and $\tau_{h}=\mathcal{I}_{h} \omega^{*}-\widetilde{\omega}_{h}$ in equation (4.15). It then follows from Proposition 2.1, Lemma 4.3, and the Cauchy-Schwarz inequality that

$$
\begin{aligned}
c_{\delta} \| & \left\{\mathcal{I}_{h} z^{*}-\widetilde{z}_{h}, \mathcal{I}_{h} \omega^{*}-\widetilde{\omega}_{h}\right\} \|_{\delta}^{2} \\
\leq & C\left(C_{\Omega}, \delta_{0}, C_{\delta}\right)\left(\left\|\left\{\mathcal{I}_{h} z^{*}-z^{*}, \mathcal{I}_{h} \omega^{*}-\omega^{*}\right\}\right\|_{\delta}+\left\|\left\{y^{*}-\widetilde{y}_{h}, \sigma^{*}-\widetilde{\sigma}_{h}\right\}\right\|_{\delta}\right) \\
& \times\left\|\left\{\mathcal{I}_{h} z^{*}-\widetilde{z}_{h}, \mathcal{I}_{h} \omega^{*}-\widetilde{\omega}_{h}\right\}\right\|_{\delta},
\end{aligned}
$$

which implies

$$
\begin{aligned}
& \left\|\left\{\mathcal{I}_{h} z^{*}-\widetilde{z}_{h}, \mathcal{I}_{h} \omega^{*}-\widetilde{\omega}_{h}\right\}\right\|_{\delta} \\
& \quad \leq C\left(\left\|\left\{\mathcal{I}_{h} z^{*}-z^{*}, \mathcal{I}_{h} \omega^{*}-\omega^{*}\right\}\right\|_{\delta}+\left\|\left\{y^{*}-\widetilde{y}_{h}, \sigma^{*}-\widetilde{\sigma}_{h}\right\}\right\|_{\delta}\right) .
\end{aligned}
$$

Then the result (4.13) can be derived by the triangle inequality, Lemma 4.3, (4.12) and (4.16); we have

$$
\begin{aligned}
\| & \left\{z^{*}-\widetilde{z}_{h}, \omega^{*}-\widetilde{\omega}_{h}\right\} \|_{\delta} \\
& \leq\left\|\left\{\mathcal{I}_{h} z^{*}-z^{*}, \mathcal{I}_{h} \omega^{*}-\omega^{*}\right\}\right\|_{\delta}+\left\|\left\{\mathcal{I}_{h} z^{*}-\widetilde{z}_{h}, \mathcal{I}_{h} \omega^{*}-\widetilde{\omega}_{h}\right\}\right\|_{\delta} \\
& \leq C\left(\left\|\left\{\mathcal{I}_{h} z^{*}-z^{*}, \mathcal{I}_{h} \omega^{*}-\omega^{*}\right\}\right\|_{\delta}+\left\|\left\{y^{*}-\widetilde{y}_{h}, \sigma^{*}-\widetilde{\sigma}_{h}\right\}\right\|_{\delta}\right) \\
& \leq C h \sum_{v=y, z ; \tau=\sigma, \omega}\left(\left\|v^{*}\right\|_{H^{2}(\Omega)}+\left\|\tau^{*}\right\|_{H^{1}(\Omega) d}\right) .
\end{aligned}
$$

Thus, Lemma 4.7 is proved.

Summarizing Lemmas 4.6-4.7 with a simple application of the triangle inequality, it is easy to obtain

$$
\begin{aligned}
\left\|\left\{y^{*}-y_{h}^{*}, \sigma^{*}-\sigma_{h}^{*}\right\}\right\|\left\|_{\delta}+\right\|\left\{z^{*}-z_{h}^{*}, \omega^{*}-\omega_{h}^{*}\right\} \|_{\delta} \\
\leq\left\|\left\{y^{*}-\widetilde{y}_{h}, \sigma^{*}-\widetilde{\sigma}_{h}\right\}\right\|_{\delta}+\left\|\left\{y_{h}^{*}-\widetilde{y}_{h}, \sigma_{h}^{*}-\widetilde{\sigma}_{h}\right\}\right\|_{\delta} \\
\quad+\left\|\left\{z^{*}-\widetilde{z}_{h}, \omega^{*}-\widetilde{\omega}_{h}\right\}\right\|_{\delta}+\left\|\left\{z_{h}^{*}-\widetilde{z}_{h}, \omega_{h}^{*}-\widetilde{\omega}_{h}\right\}\right\|_{\delta} \\
\leq C\left[\left\|u^{*}-u_{h}^{*}\right\|_{L^{2}(\Omega)}+\sum_{v=y, z ; \tau=\sigma, \omega}\left(\left\|v^{*}\right\|_{H^{2}(\Omega)}+\left\|\tau^{*}\right\|_{H^{1}(\Omega) d}\right)\right] .
\end{aligned}
$$

Therefore, the last step is to concentrate on estimating the $L^{2}$-norm errors between the continuous and discrete optimal control. 
Proof of Theorem 4.4 Note that as proved in (3.8) that $\mathcal{P}_{h} u^{*} \in U_{a d}^{h}$ for all different cases of $U_{a d}$. It then follows from the optimality conditions (2.15) and (3.7) that

$$
\begin{aligned}
& \left(\gamma u^{*}-\mathcal{B}^{*} z^{*}, u^{*}-u_{h}^{*}\right) \leq 0, \\
& \left(\gamma u_{h}^{*}-\mathcal{B}^{*} z_{h}^{*}, u_{h}^{*}-\mathcal{P}_{h} u^{*}\right) \leq 0 .
\end{aligned}
$$

Thus, we have

$$
\begin{aligned}
\gamma\left\|u^{*}-u_{h}^{*}\right\|_{L^{2}(\Omega)}^{2}= & \left(\gamma u^{*}, u^{*}-u_{h}^{*}\right)-\left(\gamma u_{h}^{*}, u^{*}-u_{h}^{*}\right) \\
= & \left(\gamma u^{*}-\mathcal{B}^{*} z^{*}, u^{*}-u_{h}^{*}\right)+\left(\gamma u_{h}^{*}-\mathcal{B}^{*} z_{h}^{*}, u_{h}^{*}-\mathcal{P}_{h} u^{*}\right) \\
& +\left(\gamma u_{h}^{*}-\mathcal{B}^{*} z_{h}^{*}, \mathcal{P}_{h} u^{*}-u^{*}\right)+\left(\mathcal{B}^{*}\left(z^{*}-z_{h}^{*}\right), u^{*}-u_{h}^{*}\right) \\
\leq & \left(\gamma u_{h}^{*}-\mathcal{B}^{*} z_{h}^{*}, \mathcal{P}_{h} u^{*}-u^{*}\right)+\left(z^{*}-z_{h}^{*}, \mathcal{B}\left(u^{*}-u_{h}^{*}\right)\right) .
\end{aligned}
$$

In the following, we try to estimate the two terms on the right-hand of (4.20). First, it follows from the definition of $\mathcal{P}_{h}$ in (3.8) that

$$
\begin{aligned}
\left(\gamma u_{h}^{*}-\mathcal{B}^{*} z_{h}^{*}, \mathcal{P}_{h} u^{*}-u^{*}\right) \\
=\left(\mathcal{B}^{*}\left(z^{*}-z_{h}^{*}\right), \mathcal{P}_{h} u^{*}-u^{*}\right)-\left(\mathcal{B}^{*} z^{*}-\mathcal{P}_{h}\left(\mathcal{B}^{*} z^{*}\right), \mathcal{P}_{h} u^{*}-u^{*}\right) \\
=\left(z^{*}-\widetilde{z}_{h}, \mathcal{B}\left(\mathcal{P}_{h} u^{*}-u^{*}\right)\right)+\left(\widetilde{z}_{h}-z_{h}^{*}, \mathcal{B}\left(\mathcal{P}_{h} u^{*}-u^{*}\right)\right) \\
\quad-\left(\mathcal{B}^{*} z^{*}-\mathcal{P}_{h}\left(\mathcal{B}^{*} z^{*}\right), \mathcal{P}_{h} u^{*}-u^{*}\right) .
\end{aligned}
$$

Thus, we conclude from the Cauchy-Schwarz inequality, the boundedness of $\mathcal{B}$, and Lemmas $4.1,4.3$ that

$$
\begin{aligned}
& \left(z^{*}-\widetilde{z}_{h}, \mathcal{B}\left(\mathcal{P}_{h} u^{*}-u^{*}\right)\right) \\
& \leq C\left\|z^{*}-\widetilde{z}_{h}\right\|_{L^{2}(\Omega)}^{2}+C\left\|\mathcal{P}_{h} u^{*}-u^{*}\right\|_{L^{2}(\Omega)}^{2} \\
& \leq C\left(C_{\Omega}, \delta_{0}\right)\left\|\left\{z^{*}-\widetilde{z}_{h}, \omega^{*}-\widetilde{\omega}_{h}\right\}\right\|_{\delta}^{2}+C\left\|\mathcal{P}_{h} u^{*}-u^{*}\right\|_{L^{2}(\Omega)}^{2} \\
& \leq C h^{2}\left[\left\|u^{*}\right\|_{H^{1}(\Omega)}^{2}+\sum_{v=y, z ; \tau=\sigma, \omega}\left(\left\|v^{*}\right\|_{H^{2}(\Omega)}^{2}+\left\|\tau^{*}\right\|_{H^{1}(\Omega)^{d}}^{2}\right)\right] \\
& \left(\widetilde{z}_{h}-z_{h}^{*}, \mathcal{B}\left(\mathcal{P}_{h} u^{*}-u^{*}\right)\right) \leq C \varepsilon\left\|\left\{z_{h}^{*}-\widetilde{z}_{h}, \omega_{h}^{*}-\widetilde{\omega}_{h}\right\}\right\|_{\delta}^{2}+C\left\|\mathcal{P}_{h} u^{*}-u^{*}\right\|_{L^{2}(\Omega)}^{2} \\
& \leq C \varepsilon\left\|u^{*}-u_{h}^{*}\right\|_{L^{2}(\Omega)}^{2}+C h^{2}\left\|u^{*}\right\|_{H^{1}(\Omega)}^{2} \\
& \left(\mathcal{B}^{*} z^{*}-\mathcal{P}_{h}\left(\mathcal{B}^{*} z^{*}\right), \mathcal{P}_{h} u^{*}-u^{*}\right) \leq C\left\|z^{*}-\mathcal{P}_{h} z^{*}\right\|_{L^{2}(\Omega)}^{2}+C\left\|\mathcal{P}_{h} u^{*}-u^{*}\right\|_{L^{2}(\Omega)}^{2} \\
& \quad \leq C h^{2}\left[\left\|u^{*}\right\|_{H^{1}(\Omega)}^{2}+\left\|z^{*}\right\|_{H^{1}(\Omega)}^{2}\right] .
\end{aligned}
$$

Here and hereafter $\varepsilon$ is a small positive constant.

Similarly, for the second term on the right-hand side of (4.20) we have

$$
\left(z^{*}-z_{h}^{*}, \mathcal{B}\left(u^{*}-u_{h}^{*}\right)\right)=\left(z^{*}-\widetilde{z}_{h}, \mathcal{B}\left(u^{*}-u_{h}^{*}\right)\right)+\left(\widetilde{z}_{h}-z_{h}^{*}, \mathcal{B}\left(u^{*}-u_{h}^{*}\right)\right),
$$


where

$$
\begin{aligned}
& \left(z^{*}-\widetilde{z}_{h}, \mathcal{B}\left(u^{*}-u_{h}^{*}\right)\right) \\
& \quad \leq C\left\|\left\{z^{*}-\widetilde{z}_{h}, \omega^{*}-\widetilde{\omega}_{h}\right\}\right\|_{\delta}^{2}+C \varepsilon\left\|u^{*}-u_{h}^{*}\right\|_{L^{2}(\Omega)}^{2} \\
& \quad \leq C \varepsilon\left\|u^{*}-u_{h}^{*}\right\|_{L^{2}(\Omega)}^{2}+C h^{2}\left[\left\|u^{*}\right\|_{H^{1}(\Omega)}^{2}+\sum_{v=y, z ; \tau=\sigma, \omega}\left(\left\|v^{*}\right\|_{H^{2}(\Omega)}^{2}+\left\|\tau^{*}\right\|_{H^{1}(\Omega) d}^{2}\right)\right],
\end{aligned}
$$

and following (4.8) and (4.10) we have

$$
\begin{aligned}
\left(\widetilde{z}_{h}-z_{h}^{*}, \mathcal{B}\left(u^{*}-u_{h}^{*}\right)\right) & =\mathcal{A}_{\delta}\left(y_{h}^{*}-\tilde{y}_{h}, \sigma_{h}^{*}-\widetilde{\sigma}_{h} ; z_{h}^{*}-\widetilde{z}_{h}, \omega_{h}^{*}-\widetilde{\omega}_{h}\right) \\
& =-\left(y_{h}^{*}-\tilde{y}_{h}, y_{h}^{*}-\tilde{y}_{h}\right)-\left(\sigma_{h}^{*}-\widetilde{\sigma}_{h}, \sigma_{h}^{*}-\widetilde{\sigma}_{h}\right) \\
& \leq 0 .
\end{aligned}
$$

Finally, collecting all these bounds together with $\varepsilon$ being small enough, we have

$$
\left\|u^{*}-u_{h}^{*}\right\|_{L^{2}(\Omega)} \leq C h\left[\left\|u^{*}\right\|_{H^{1}(\Omega)}+\sum_{v=y, z ; \tau=\sigma, \omega}\left(\left\|v^{*}\right\|_{H^{2}(\Omega)}+\left\|\tau^{*}\right\|_{H^{1}(\Omega)}\right)\right] .
$$

Furthermore, inserting the above estimate into (4.18) we directly have

$$
\begin{aligned}
& \left\|\left\{y^{*}-y_{h}^{*}, \sigma^{*}-\sigma_{h}^{*}\right\}\right\|_{\delta}+\left\|\left\{z^{*}-z_{h}^{*}, \omega^{*}-\omega_{h}^{*}\right\}\right\|_{\delta} \\
& \quad \leq C h\left[\left\|u^{*}\right\|_{H^{1}(\Omega)}+\sum_{v=y, z ; \tau=\sigma, \omega}\left(\left\|v^{*}\right\|_{H^{2}(\Omega)}+\left\|\tau^{*}\right\|_{H^{1}(\Omega)^{d}}\right)\right] .
\end{aligned}
$$

Thus, Theorem 4.4 follows immediately from (4.23) and (4.24).

\section{Numerical experiments}

In this section, we present some numerical results of the novel stabilized mixed finite element method defined in Section 3 which confirm the theoretical analysis of the previous section. As for the constrained optimal control problem, the states and control are the main concern of the practical problem. Therefore, in the following tests, we mostly focus on the results of the state $y$, the flux state $\sigma$, and the control $u$.

Let $\Omega=[0,1] \times[0,1]$. We are ready to test the following type optimal control problem:

$$
\min _{u \in U_{a d}} \frac{1}{2} \int_{\Omega}\left(y-y_{d}\right)^{2}+\frac{1}{2} \int_{\Omega}\left(\sigma-\sigma_{d}\right)^{2}+\frac{1}{2} \int_{\Omega} u^{2}
$$

subject to

$$
\begin{cases}-\Delta y=f+u, & \text { in } \Omega, \\ \sigma=-\nabla y, & \text { in } \Omega .\end{cases}
$$

For simplicity, we choose $\delta=0.8$ as the stabilized parameter. Furthermore, to solve the constrained optimal control problem numerically, we adopt the following iterations using a Matlab environment. 


\section{Algorithm}

Step 1. Give an initial control solution $u_{h}^{(0)} \in U_{a d}^{h}$ and tolerance TOL $=1.0 \mathrm{E}-06$.

For $n=1,2, \ldots, \operatorname{Nmax}=100$

Step 2. Solve $\left(y_{h}^{(n)}, \sigma_{h}^{(n)}\right) \in Y^{h} \times \Sigma^{h}$ as follows:

Step 2.1. Given initial $\sigma_{h}^{(n, 0)}$ and tolerance TOL ${ }^{\prime}=1.0 \mathrm{E}-03$.

For $k=1,2, \ldots, \operatorname{Nmax}^{\prime}=20$

Step 2.2. Solve $y_{h}^{(n, k)} \in Y^{h}$ such that

$$
\delta\left(\nabla y_{h}^{(n, k)}, \nabla v_{h}\right)=\left(f+u_{h}^{(n-1)}, v_{h}\right)+(1-\delta)\left(\sigma_{h}^{(n, k-1)}, \nabla v_{h}\right) .
$$

Step 2.3. Solve $\sigma_{h}^{(n, k)} \in \Sigma^{h}$ such that

$$
\left(\sigma_{h}^{(n, k)}, \tau_{h}\right)=-\left(\nabla y_{h}^{(n, k)}, \tau_{h}\right)
$$

Step 2.4. Stop until $\left\|\sigma_{h}^{(n, k)}-\sigma_{h}^{(n, k-1)}\right\|_{L^{2}(\Omega)^{2}}<\mathrm{TOL}^{\prime}$ is satisfied, and let

$$
y_{h}^{(n)}=y_{h}^{(n, k)}, \quad \sigma_{h}^{(n)}=\sigma_{h}^{(n, k)} .
$$

Step 3. Solve $\left(z_{h}^{(n)}, \omega_{h}^{(n)}\right) \in Y^{h} \times \Sigma^{h}$ as follows:

Step 3.1. Given initial $\omega_{h}^{(n, 0)}$ and tolerance TOL'.

For $k=1,2, \ldots, \mathrm{Nmax}^{\prime}$

Step 3.2. Solve $z_{h}^{(n, k)} \in Y^{h}$ such that

$$
\delta\left(\nabla z_{h}^{(n, k)}, \nabla v_{h}\right)=-\left(y_{h}^{(n)}-y_{d}, v_{h}\right)-(1-\delta)\left(\omega_{h}^{(n, k-1)}, \nabla v_{h}\right) .
$$

Step 3.3. Solve $\omega_{h}^{(n, k)} \in \Sigma^{h}$ such that

$$
\left(\omega_{h}^{(n, k)}, \tau_{h}\right)=\left(\nabla z_{h}^{(n, k)}, \tau_{h}\right)-\frac{1}{1-\delta}\left(\sigma_{h}^{(n)}-\sigma_{d}, \tau_{h}\right) .
$$

Step 3.4. Stop until $\left\|\omega_{h}^{(n, k)}-\omega_{h}^{(n, k-1)}\right\|_{L^{2}(\Omega)^{2}}<\mathrm{TOL}^{\prime}$ is satisfied, and let

$$
z_{h}^{(n)}=z_{h}^{(n, k)}, \quad \omega_{h}^{(n)}=\omega_{h}^{(n, k)} .
$$

Step 4. Solve $u_{h}^{(n)} \in U_{a d}^{h}$ such that

$$
\left(u_{h}^{(n)}-z_{h}^{(n)}, v_{h}-u_{h}^{(n)}\right) \geq 0 .
$$

Step 5. Stop if $\left\|u_{h}^{(n)}-u_{h}^{(n-1)}\right\|_{L^{2}(\Omega)}<$ TOL is satisfied, and let

$$
u_{h}=u_{h}^{(n)}, \quad y_{h}=y_{h}^{(n)}, \quad \sigma_{h}=\sigma_{h}^{(n)}, \quad z_{h}=z_{h}^{(n)}, \quad \omega_{h}=\omega_{h}^{(n)} .
$$

Example 1 For the first example, we choose $\sigma_{d}=\sigma$ and consider Case III with $a=0, b=$ 0.5 . The corresponding analytical solutions of the optimal control problem are as follows:

$$
y(x)=\sin \left(\pi x_{1}\right) \sin \left(\pi x_{2}\right)
$$


Table 1 Errors, convergence orders and CPU time for Example 1

\begin{tabular}{lllllc}
\hline Mesh & $\| \boldsymbol{u}-\boldsymbol{u}_{\boldsymbol{h}} \boldsymbol{\|}_{\mathbf{L}^{\mathbf{2}} \boldsymbol{( \Omega )}}$ & Order & \|\| $\boldsymbol{y}-\boldsymbol{y}_{\boldsymbol{h}}, \boldsymbol{\sigma}-\boldsymbol{\sigma}_{\boldsymbol{h}} \boldsymbol{\}} \|_{\boldsymbol{\delta}}$ & Order & CPU time \\
\hline $8^{*} 8$ & $5.5232 \mathrm{E}-02$ & - & $4.0521 \mathrm{E}-01$ & - & $5 \mathrm{~s}$ \\
$16^{*} 16$ & $2.7731 \mathrm{E}-02$ & 0.99 & $1.9822 \mathrm{E}-01$ & 1.03 & $26 \mathrm{~s}$ \\
$32^{*} 32$ & $1.3741 \mathrm{E}-02$ & 1.01 & $9.8230 \mathrm{E}-02$ & 1.01 & $127 \mathrm{~s}$ \\
$64^{*} 64$ & $6.9009 \mathrm{E}-03$ & 0.99 & $4.8929 \mathrm{E}-02$ & 1.01 & $761 \mathrm{~s}$ \\
\hline
\end{tabular}
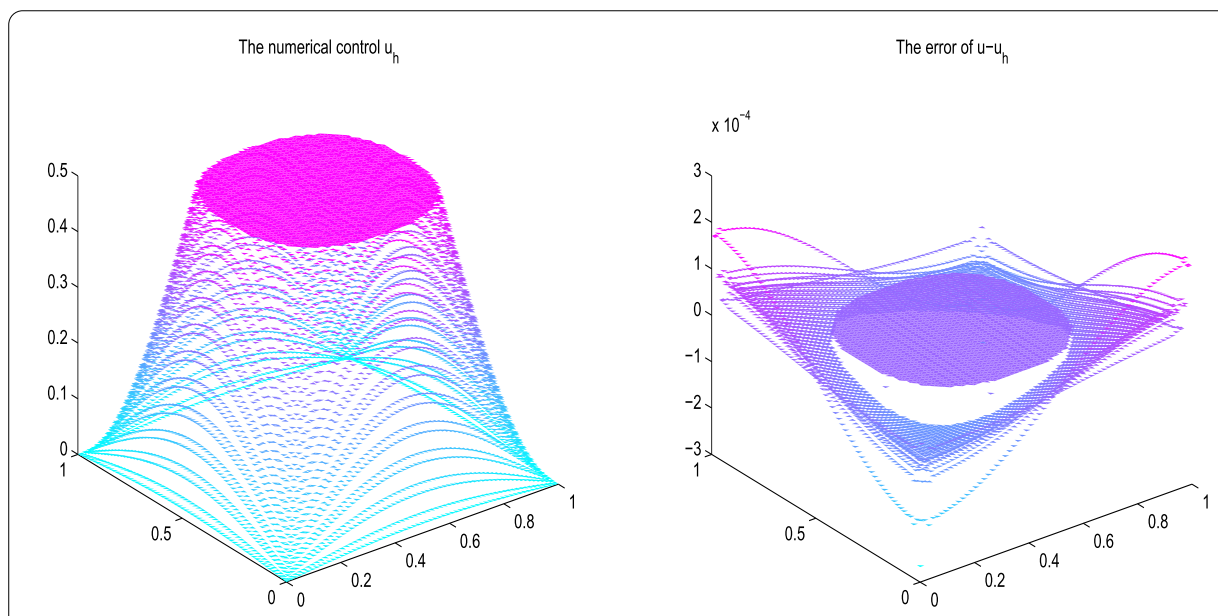

Figure 1 The numerical control $u_{h}$ and corresponding error for Example 1.
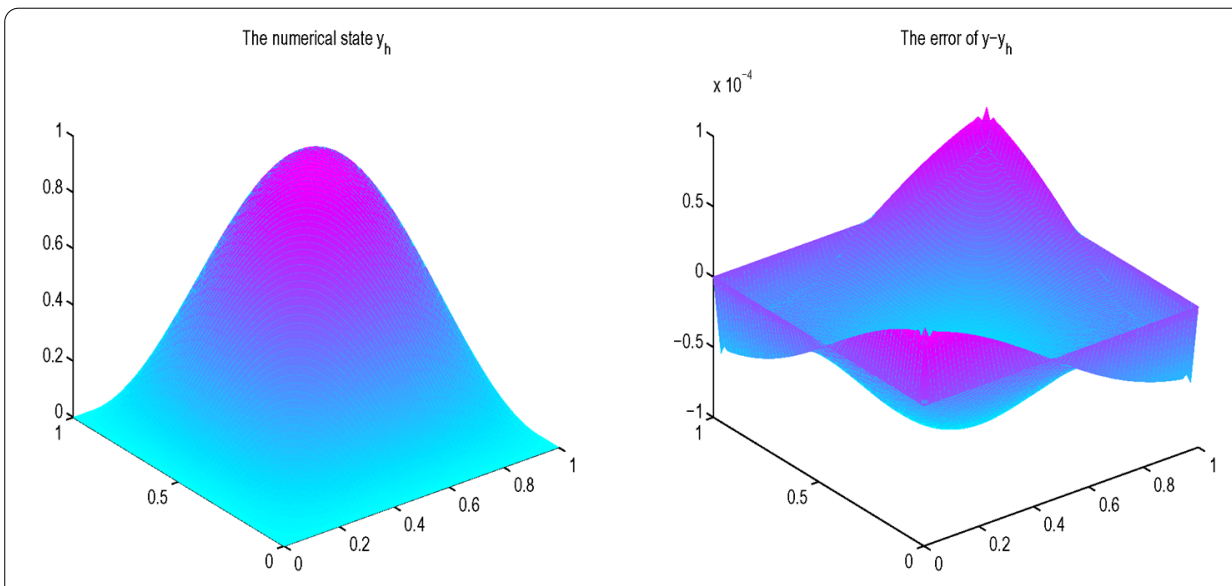

Figure 2 The numerical state $y_{h}$ and corresponding error for Example 1.

$$
\begin{aligned}
& \sigma(x)=-\nabla y, \\
& z(x)=\sin \left(\pi x_{1}\right) \sin \left(\pi x_{2}\right), \\
& \omega(x)=\nabla z, \\
& u(x)=\max \{0, \min \{0.5, z\},
\end{aligned}
$$

where the source function $f$ can be computed using (1.2), while the desired state $y_{d}$ is determined by (2.16). 

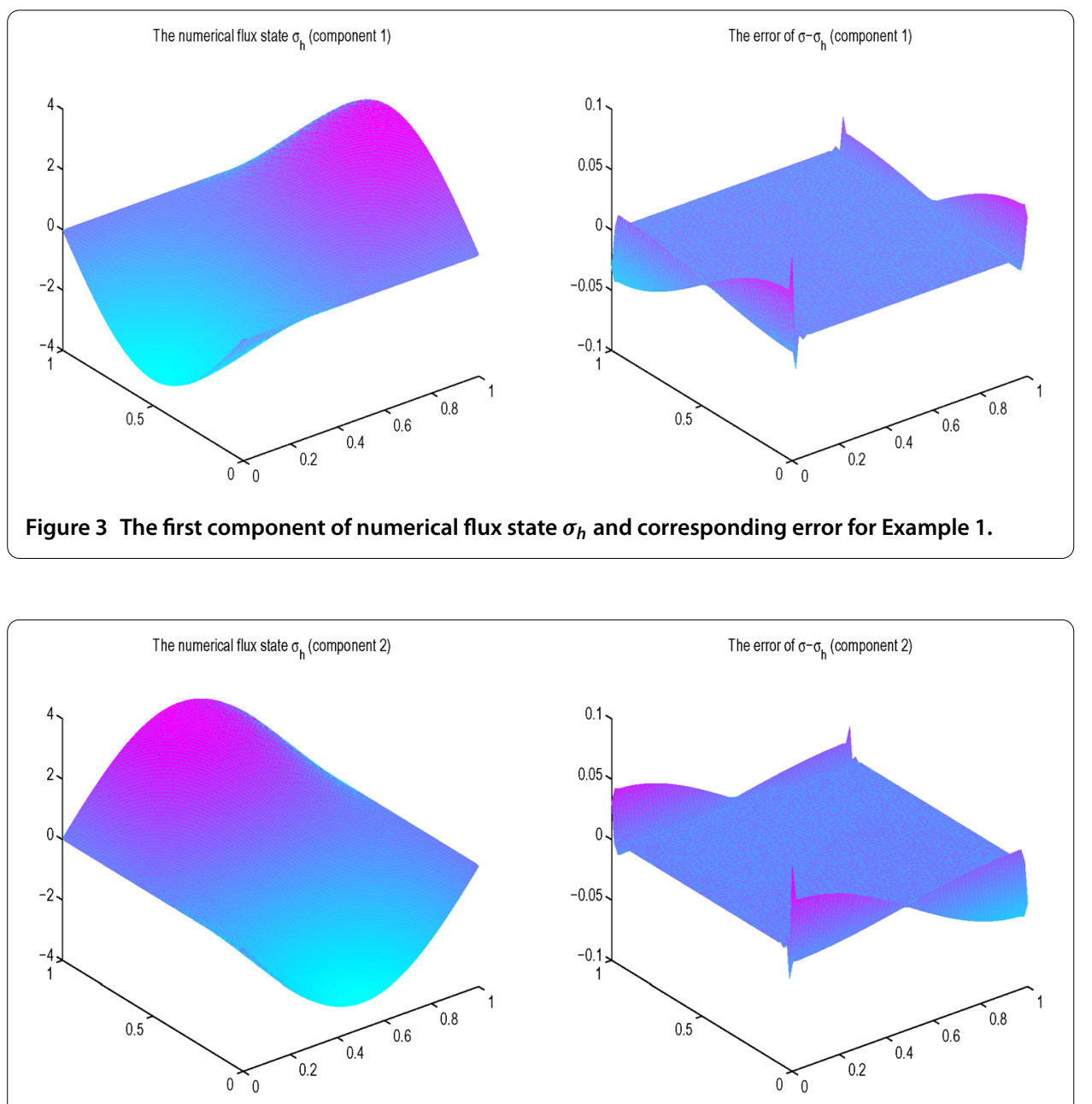

Figure 4 The second component of numerical flux state $\sigma_{h}$ and corresponding error for Example 1.

We can see that the solutions of this example are $\delta$-independent. Table 1 displays the errors and convergence orders with respect to the decreasing uniform mesh size $h$ for the control $u$ in $L^{2}(\Omega)$-norm, the states $y$ and $\sigma$ in weighted norm. The main CPU time for the computation excluding the mesh generation part is also listed. It uses no more than five cycles for the iteration algorithm. Figure 1 shows the numerical solution and numerical error of the control when mesh size $h=1 / 64$, while the numerical solutions and errors of the state and flux state are presented in Figures 2-4, respectively. It can be observed that the numerical results are in agreement with the analytical solutions very well.

Example 2 For the second example, we consider a nonhomogeneous Dirichlet boundary problem. Let us take $\sigma_{d}=0$ and consider Case IV with $\bar{z}=\frac{4}{9}$. The corresponding solutions of the optimal control problem are as follows:

$$
\begin{aligned}
& y(x)=x_{1}\left(1-x_{1}\right)+x_{2}\left(1-x_{2}\right), \\
& \sigma(x)=-\nabla y, \\
& z(x)=16 x_{1}\left(1-x_{1}\right) x_{2}\left(1-x_{2}\right),
\end{aligned}
$$


Table 2 Errors, convergence orders and CPU time for Example 2

\begin{tabular}{lllllc}
\hline Mesh & $\| \boldsymbol{u}-\boldsymbol{u}_{\boldsymbol{h}} \boldsymbol{\|}_{\mathbf{L}^{\mathbf{2}(\boldsymbol{\Omega})}}$ & Order & $\| \boldsymbol{\|} \boldsymbol{y}-\boldsymbol{y}_{\boldsymbol{h}}, \boldsymbol{\sigma}-\boldsymbol{\sigma}_{\boldsymbol{h}} \boldsymbol{\}} \boldsymbol{\| l}_{\boldsymbol{\delta}}$ & Order & CPU time \\
\hline $8^{*} 8$ & $7.1264 \mathrm{E}-02$ & - & $9.5403 \mathrm{E}-02$ & - & $8 \mathrm{~s}$ \\
$16^{*} 16$ & $3.5255 \mathrm{E}-02$ & 1.02 & $4.6587 \mathrm{E}-02$ & 1.03 & $37 \mathrm{~s}$ \\
$32^{*} 32$ & $1.7583 \mathrm{E}-02$ & 1.00 & $2.3046 \mathrm{E}-02$ & 1.02 & $172 \mathrm{~s}$ \\
$64^{*} 64$ & $8.7859 \mathrm{E}-03$ & 1.00 & $1.1465 \mathrm{E}-02$ & 1.01 & $973 \mathrm{~s}$ \\
\hline
\end{tabular}
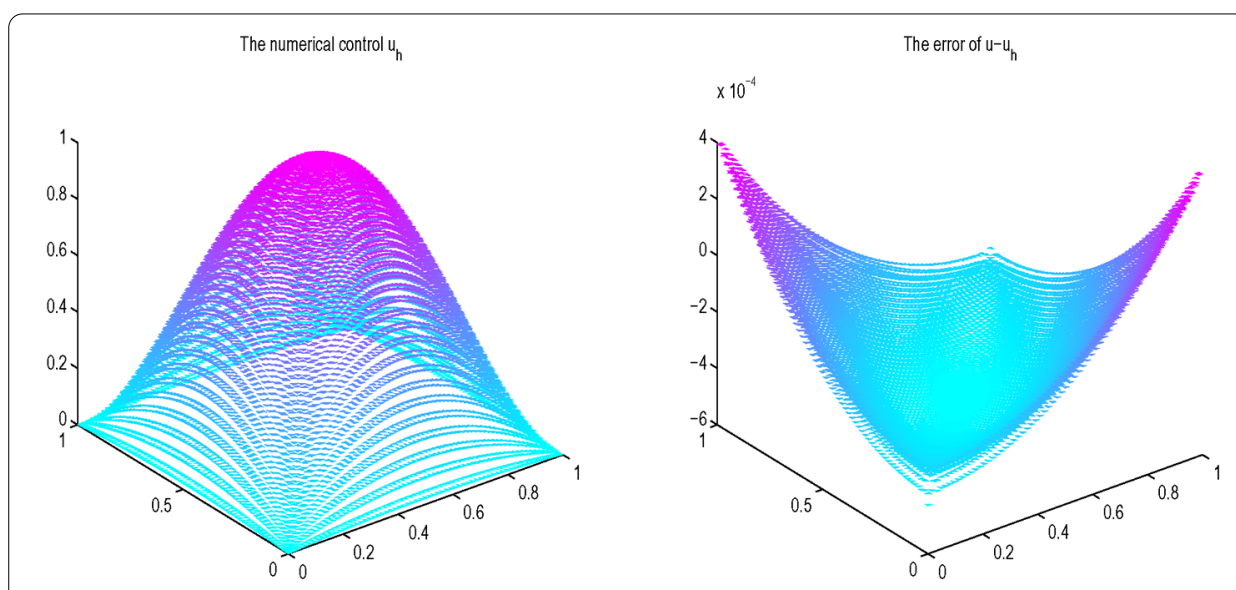

Figure 5 The numerical control $u_{h}$ and corresponding error for Example 2.
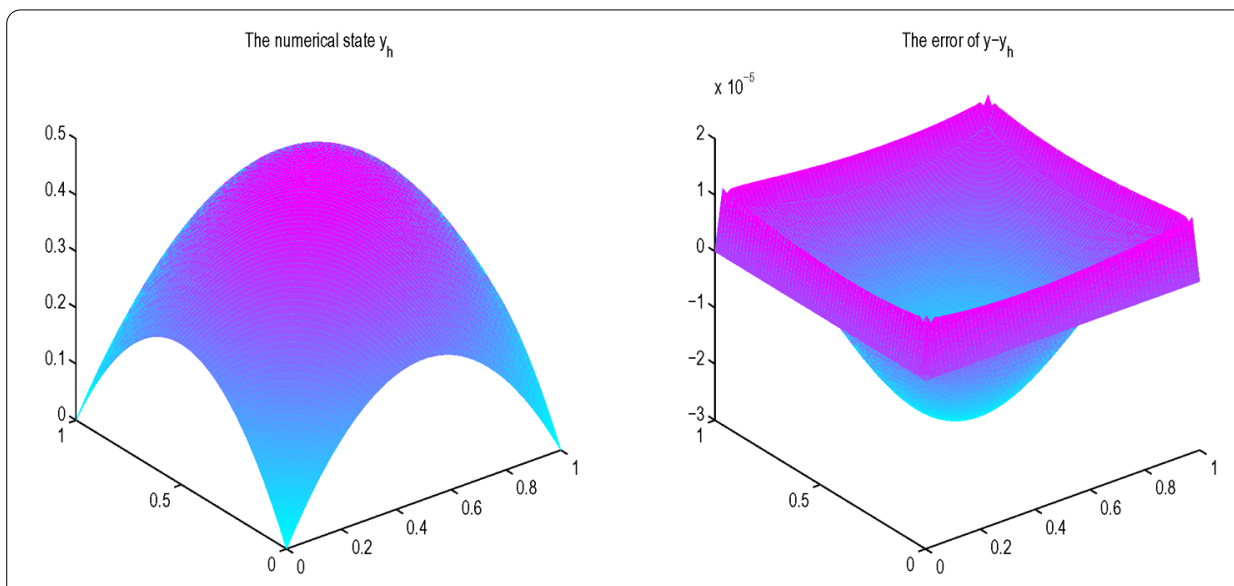

Figure 6 The numerical state $y_{h}$ and corresponding error for Example 2.

$$
\begin{aligned}
& \omega(x)=\nabla z-\frac{\sigma-\sigma_{d}}{1-\delta}, \\
& u(x)=z
\end{aligned}
$$

where the source function $f$ and the desired states $y_{d}$ can also be determined by (1.2) and (2.16), respectively.

This is a $\delta$-dependent example, i.e., the adjoint flux state $\omega$ depends on the parameter $\delta$. In Table 2, we also list the corresponding errors, convergence orders and CPU time with respect to different meshes. Figures 5-8 show the plots of the numerical control, state, and flux state, respectively for $h=1 / 64$. We can observe that the convergence orders are 


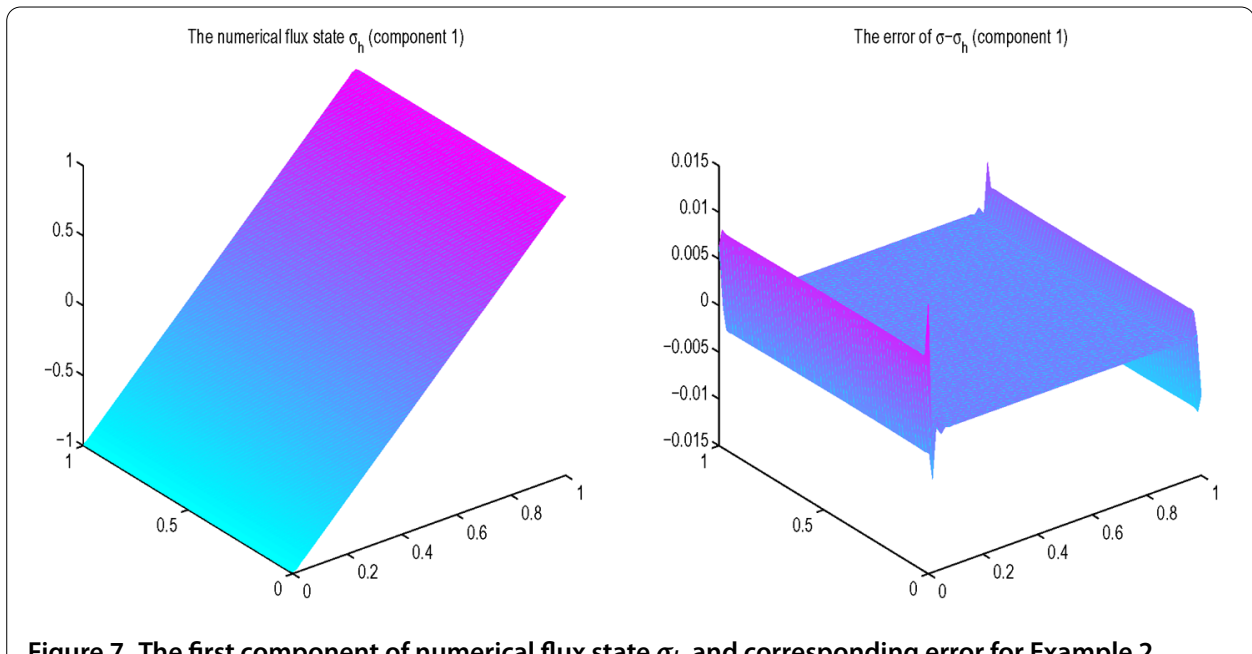

Figure 7 The first component of numerical flux state $\sigma_{h}$ and corresponding error for Example 2.
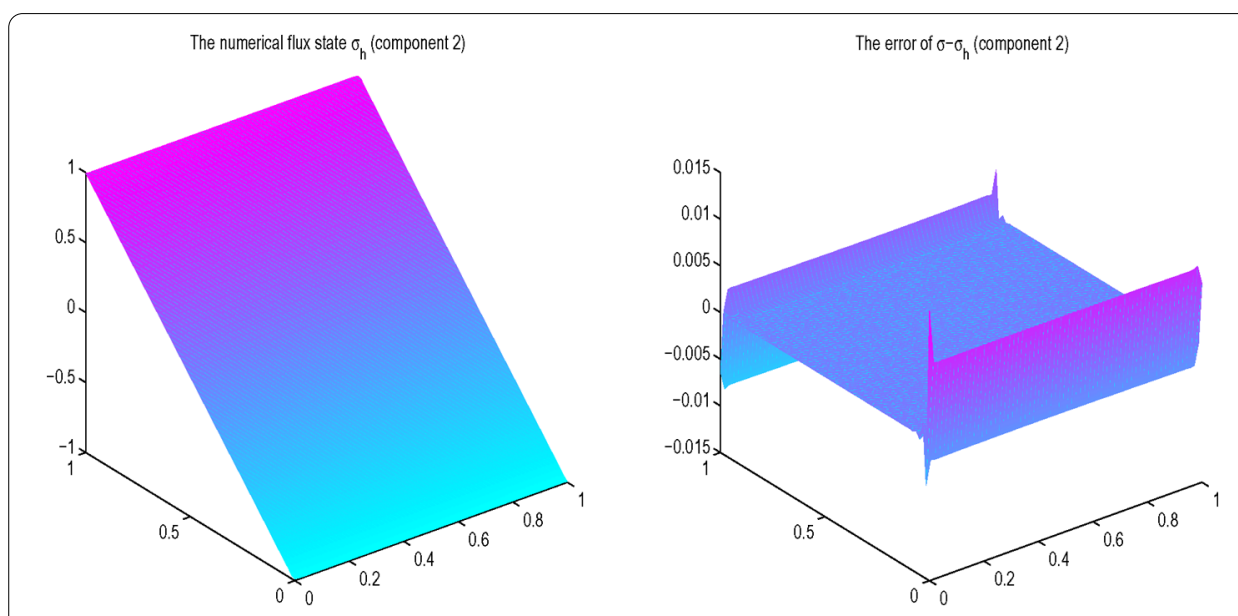

Figure 8 The second component of numerical flux state $\sigma_{h}$ and corresponding error for Example 2 .

consistent with Theorem 4.4. Besides, the numerical results are also well matched with the analytical solutions.

\section{Concluding remarks}

In this paper, we discuss a novel stabilized mixed finite element method for the approximation of reaction-diffusion optimal control problem. Compared with our previous work, the novel method is more simple and easier to be implemented. It needs only one stabilization parameter but is still stable. Furthermore, low regularities for the state and adjoint state variables are needed. Different cases of the admissible control set are discussed and a priori error estimates are proved. Finally, numerical experiments are addressed to demonstrate the theoretical analysis. Most importantly, we should point that this novel stabilized mixed method is more competitive. It can easily be extended to solve optimal control problems governed by a bilinear state equation, the Stokes equation and so on. 
Authors' contributions

All authors read and approved the final manuscript.

\section{Author details}

${ }^{1}$ College of Science, China University of Petroleum, Qingdao, 266580, China. ${ }^{2}$ College of Petroleum Engineering, China University of Petroleum, Qingdao, 266580, China.

\section{Acknowledgements}

This work was supported by the National Natural Science Foundation of China (Nos. 11201485, 11571367), the Promotive Research Fund for Excellent Young and Middle-aged Scientists of Shandong Province (No. BS2013NJ001), and the Fundamental Research Funds for the Central Universities (Nos. 14CX02217A, 15CX08004A, 15CX08011A). The first author was partially supported by the China Scholarship Council (No. 201506455014).

Received: 3 October 2015 Accepted: 12 January 2016 Published online: 26 January 2016

\section{References}

1. Ciarlet, PG: The Finite Element Method for Elliptic Problems. SIAM, Philadelphia (2002)

2. Brenner, SC, Scott, LR: The Mathematical Theory of Finite Element Methods. Springer, New York (2010)

3. Lions, JL: Optimal Control of Systems Governed by Partial Differential Equations. Springer, Berlin (1971)

4. Liu, W, Yan, N: Adaptive Finite Element Methods for Optimal Control Governed by PDEs. Series in Information and Computational Science, vol. 41. Science Press, Beijing (2008)

5. Tiba, D: Lectures on the Optimal Control of Elliptic Equations. University of Jyväskylä Press, Jyväskylä (1995)

6. Hinze, M, Pinnau, R, Ulbrich, M, Ulbrich, S: Optimization with PDE Constraints. Springer, Berlin (2009)

7. Falk, FS: Approximation of a class of optimal control problems with order of convergence estimates. J. Math. Anal. Appl. 44, 28-47 (1973)

8. Hinze, M: A variational discretization concept in control constrained optimization: the linear-quadratic case. Comput. Optim. Appl. 30, 45-61 (2005)

9. Meyer, C, Rösch, A: Superconvergence properties of optimal control problems. SIAM J. Control Optim. 43, 970-985 (2004)

10. Arada, N, Casas, E, Tröltzsch, F: Error estimates for the numerical approximation of a semilinear elliptic control problem. Comput. Optim. Appl. 23, 201-229 (2002)

11. Chen, Y, Liu, W: Error estimates and superconvergence of mixed finite elements for quadratic optimal control. Int. J. Numer. Anal. Model. 3, 311-321 (2006)

12. Yan, N, Zhou, Z: A RT mixed FEM/DG scheme for optimal control governed by convection diffusion equations. J. Sci. Comput. 41, 273-299 (2009)

13. Chen, Y, Lu, Z: Error estimates of fully discrete mixed finite element methods for semilinear quadratic parabolic optimal control problem. Comput. Methods Appl. Mech. Eng. 199, 1415-1423 (2010)

14. Zhou, J, Chen, Y, Dai, Y: Superconvergence of triangular mixed finite elements for optimal control problems with an integral constraint. Appl. Math. Comput. 217, 2057-2066 (2010)

15. Fu, H, Rui, H: A characteristic-mixed finite element method for time-dependent convection-diffusion optimal control problem. Appl. Math. Comput. 218, 3430-3440 (2011)

16. Gong, W, Yan, N: Mixed finite element method for Dirichlet boundary control problems governed by elliptic PDEs. SIAM J. Control Optim. 49, 984-1014 (2011)

17. Raviart, PA, Thomas, JM: A mixed finite element method for 2 nd order elliptic problems. In: Mathematical Aspects of Finite Element Methods. Lecture Notes in Mathematics, vol. 606, pp. 292-315. Springer, Berlin (1977)

18. Hughes, TJR, Franca, LP, Hulbert, G: A new finite element formulation for computational fluid dynamics: VIII. The Galerkin/least-squares method for advective-diffusive equations. Comput. Methods Appl. Mech. Eng. 73, 173-189 (1989)

19. Franca, LP, Stenberg, R: Error analysis of Galerkin least squares methods for the elasticity equations. SIAM J. Numer Anal. 28, 1680-1697 (1991)

20. Franca, LP, Frey, SL, Hughes, TJR: Stabilized finite element methods: I. Application to the advective-diffusive model. Comput. Methods Appl. Mech. Eng. 95, 253-276 (1992)

21. Franca, LP, Valentin, F: On an improved unusual stabilized finite element method for the advective-reactive-diffusive equation. Comput. Methods Appl. Mech. Eng. 190, 1785-1800 (2000)

22. Fu, H, Rui, H, Hou, J, Li, H: A stabilized mixed finite element method for elliptic optimal control problems. J. Sci. Comput. (2015). doi:10.1007/s10915-015-0050-3.5

23. Brezzi, F, Fortin, M: Mixed and Hybrid Finite Element Methods. Springer, New York (1991) 